SFB

On- and offline detection of

823

\title{
structural breaks in thermal
} spraying processes

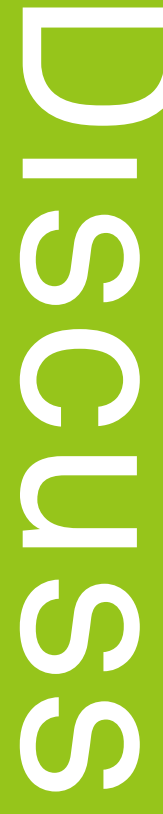

Matthias Borowski, Nikolaus Rudak,

Birger Hussong, Dominik Wied, Sonja Kuhnt, Wolfgang Tillmann

\section{Nr. 2/2013}

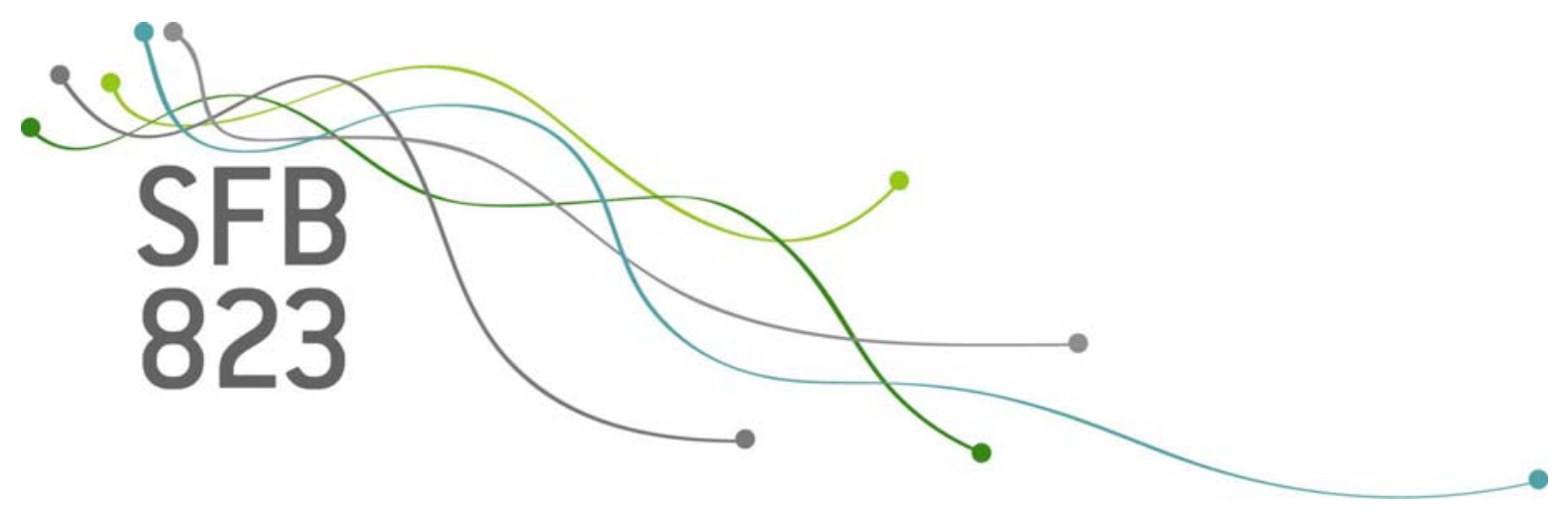





\title{
On- and Offline Detection of Structural Breaks in Thermal Spraying Processes
}

\author{
Matthias Borowski* Nikolaus Rudak* Birger Hussong* \\ Dominik Wied* Sonja Kuhnt* Wolfgang Tillmann* \\ January 23, 2013
}

\begin{abstract}
The detection of structural changes in time series from industrial processes monitoring is of great interest. We investigate the limits and possibilities of two recently developed methods, one for the off-line detection of changed volatilities [Wied et al., 2011 ] and one for the on-line detection of changes in the course of the time series [Borowski and Fried, 2011]. The investigation is carried out on several time series from thermal spraying processes. The processes are deliberately manipulated to produce structural changes at known time points.
\end{abstract}

\section{Introduction}

Industrial production processes are often supervised by the ongoing measurement of several variables. The reliable and fast detection of changes in the resulting time series can help to avoid production failures and is therefore of great interest.

The quality of thermally sprayed coatings as an an example depends on a variety of factors. Some factors like the spray distance, path strategy and gun velocity can be easily adjusted and controlled. Other factors like the mass-flow of the process gases, pressure in the combustion chamber and flow of the feedstock powder have to be controlled electronically. Irregularities

*TU Dortmund University 
affecting the coating result are observable in the particle properties of the spray jet. Hence, the time series of measured particle properties yield valuable information which can be obtained by suitable statistical methods. An offline analysis of these time series is useful to assign the quality of the coating after the spraying process has finished and all data have been observed. In an online analysis the spraying process (and hence the coating process) is monitored in realtime, i.e. the time series are treated as data streams. If irregularities are detected online, the engineer has the opportunity to adjust the relevant factors and thus to assure the quality of the coating. The on- and offline detection of irregularities are flexible approaches applicable to any kind of spraying system regardless of which other sensors or control mechanisms are given. Scientists in engineering, mathematics and statistics of the collaborative research center 823 of the Deutsche Forschungsgemeinschaft develop new procedures for the on- and offline analysis of time series. We consider recently developed methods useful for the offline detection of changes in the variance [Wied et al., 2011] and the online detection of changes in the assumed underlying signal [Borowski and Fried, 2011]. These methods can be used independently of each other and can therefore be combined in order to get a powerful controling tool. In this article, the methods are applied to time series of variables measured during a coating process to investigate what kind of change points can be detected during the process. These findings will be usefull for future spray process monitoring systems and might even be applied to sensors on the tips of a spray gun.

\section{Experimental Set-Up}

The experiments were conducted using an HVOF gun of type WokaJet 400 from Sulzer Metco with a MultiCoat control module. Using this module allows a continuous electronic monitoring of all primary system parameters. A TWIN 120AH powder feeder provided a steady powder supply to the hot gas jet by two radial powder injectors. An agglomerated and sintered WC-Co powder with particle sizes between 15 and $45 \mu \mathrm{m}$ was chosen as feedstock. For all spray plume measurements an AccuraSpray g3 system from Tecnar was employed.

For the simulation of process malfunctions, several experiments were run with standardized start parameters suitable for the feedstock. After 7 minutes of measuring time, the spray parameters were altered according to the malfunction to be simulated. Another 7 minutes measuring time with the altered parameters should give enough data for statistical analysis of the structural 
interruptions. We consider four different interruption types. Firstly, we enforce the failure of one powder feeder by switching off the feeder disc on one side only. This manipulation does not interrupt the process immediately due to the well working second powder feeder depletion. Secondly, we lower the carrier gas pressure by $5 \%$ which leads to a pressure drop in the carrier gas. Thirdly, we switch off the compressed air supply for the AccuraySpray g3 system which measures the in-flight particle properties. This causes a breakdown of the Accuraspray air cooling. Fourthly, in opposite to the first three types of process interruption, the nozzle wear could not be investigated in one single run. Due to the fact, that nozzle wear proceeds slowly, it is impossible to run the machines until the normal wear exceeds a reasonable level. As the progress of wear is a steady function, it will not show any structural interruptions. Therefore the mathematical analysis of this factor does not focus on time-dependent changes, but on general differences in the structure of the collected data when a new or an old nozzle is mounted to the spray gun. Table 1 shows the different possible process interruptions and the corresponding procedure to simulate them.

\begin{tabular}{l|l}
\hline Interruption type & Simulation procedure \\
\hline $\begin{array}{l}\text { Failure of one powder } \\
\text { feeder depletion }\end{array}$ & $\begin{array}{l}\text { Switching off feeder disc } \\
\text { on one side only }\end{array}$ \\
\hline $\begin{array}{l}\text { Pressure drop of } \\
\text { the carrier gas }\end{array}$ & $\begin{array}{l}\text { Lowering the carrier } \\
\text { gas pressure by } 5 \%\end{array}$ \\
\hline $\begin{array}{l}\text { breakdown of the } \\
\text { AccuraSpray air cooling }\end{array}$ & $\begin{array}{l}\text { Switching off the compressed } \\
\text { air supply for the AccuraSpray g3 }\end{array}$ \\
\hline critical wear of the & $\begin{array}{l}\text { Replacement of new nozzle } \\
\text { by an old one showing } \\
\text { acceleration nozzle }\end{array}$ \\
& $\begin{array}{l}\text { excessive wear } \\
\text { (machine stop unavoidable) }\end{array}$ \\
\hline
\end{tabular}

Table 1: Possible process interruptions and the corresponding procedure

The AccuraSpray g3 provides a bunch of different types of data, which could be interpreted. Four of these values were chosen for mathematical interpretation: The particle velocities, particle temperatures, spray jet width and intensity. Besides the mean values of these quantities, the system also records all standard deviations for each second. While analyzing the influence of a breakdown of the AccuraSpray air cooling, the values for current measurement headtemperature was correlated to the spray jet data as well. 


\section{Fluctuation test for constant variances}

A recently developed fluctuation test for constant variances originally introduced by Wied et al. [2011] is presented in this section. The test problem with the test statistic is introduced followed by the distribution of the null hypothesis which is a result from Wied et al. [2011]. Afterwards it is applied to the data coming from the thermal spraying process.

\subsection{Test statistic and properties}

In the following we describe the fluctuation test. For a sequence of random variables, namely $\left(X_{t}, t=1,2, \ldots\right)$, which are assumed to have finite absolute $(4+\delta)$ th moments $(\delta \in \mathbb{R})$ our focus is to test whether the variance is constant over time. Therefore we seek to investigate the following test problem

$$
H_{0}: \operatorname{Var}\left(X_{1}\right)=\ldots=\operatorname{Var}\left(X_{T}\right) \text { vs. } H_{1}: \exists t \in\{1, \ldots, T-1\}: \operatorname{Var}\left(X_{t}\right) \neq \operatorname{Var}\left(X_{t+1}\right) .
$$

The corresponding test statistic is

$$
Q_{T}(X)=\max _{1 \leq j \leq T}\left|\hat{D} \frac{j}{\sqrt{T}}\left([\operatorname{Var} X]_{j}-[\operatorname{Var} X]_{T}\right)\right|
$$

where

$$
[\operatorname{Var} X]_{l}=\frac{1}{l} \sum_{t=1}^{l} X_{t}^{2}-\left(\frac{1}{l} \sum_{t=1}^{l} X_{t}\right)^{2}=: \overline{X_{l}^{2}}-\left(\bar{X}_{l}\right)^{2}
$$

is the empirical variance calculated from the first 1 observations. Furthermore we have

$$
\hat{D}=\left(\left(1,-2 \overline{X_{T}}\right) \hat{D}_{1}\left(1,-2 \overline{X_{T}}\right)^{\prime}\right)^{-1 / 2}
$$

with

$$
\hat{D}_{1}=\frac{1}{T} \sum_{t=1}^{T} \hat{U}_{t} \hat{U}_{t}^{\prime}+2 \sum_{j=1}^{T} k\left(\frac{j}{\gamma_{T}}\right) \frac{1}{T} \sum_{t=1}^{T-j} \hat{U}_{t} \hat{U}_{t+j}^{\prime}
$$

and

$$
\begin{aligned}
\hat{U}_{l} & =\left(\begin{array}{l}
X_{l}^{2}-\overline{X_{T}^{2}} \\
X_{l}-\overline{X_{T}}
\end{array}\right), \\
k(x) & = \begin{cases}1-|x|, & |x| \leq 1 \\
0, & \text { otherwise }\end{cases} \\
\gamma_{T} & =\sqrt{T} .
\end{aligned}
$$


The test rejects the null hypothesis if the empirical variances fluctuate too much over time.

In order to derive a limiting null distribution the following assumptions have to be stated, compare Wied et al. [2011].

(A1) The sequence $\left(X_{t}, t=1,2, \ldots\right)$ is weak-sense stationary.

(A2) For

$$
U_{t}=\left(\begin{array}{c}
X_{t}^{2}-E\left(X_{1}^{2}\right) \\
X_{t}-E\left(X_{1}\right)
\end{array}\right)
$$

and $S_{j}=\sum_{t=1}^{j}$, we have

$$
\lim _{T \rightarrow \infty} E\left(\frac{1}{T} S_{T} S_{T}^{\prime}\right)=: D_{1} \text { is finite and positive definite. }
$$

(A3) The r-th absolute value of the components of $U_{t}$ are uniformly bounded for some $r>2$.

(A4) The sequence $\left(X_{t}, t=1,2, \ldots\right)$ is $L_{2}$-NED (near-epoch dependent) with size $-\frac{r-1}{r-2}$, with $\mathrm{r}$ from $(\mathrm{A} 3)$, and constants $\left(c_{t}\right), t=1,2, \ldots$ on a sequence $\left(V_{t}\right), t=1,2, \ldots$ which is $\alpha$-mixing of size $\phi^{*}:=-\frac{r}{r-2}$, such that

$$
c_{t} \leq 2\left(\left\{E\left|X_{t}^{2}-E\left(X_{1}^{2}\right)\right|^{2}+E\left|X_{t}-E\left(X_{1}\right)\right|^{2}\right\}\right)^{\frac{1}{2}} .
$$

The limiting null distribution of the test statistic $Q_{T}$ can now be stated in the following theorem, see Wied et al. [2011].

Theorem 3.1 Under $H_{0}$ and assumptions (A1) to (A4) we have

$$
Q_{T}(X) \rightarrow \sup _{z \in[0,1]}|B(z)|,
$$

where $B(z)$ is a one dimensional Brownian bridge.

Theorem 3.1 allows to determine quantiles of the null distribution in order to apply the test. The quantiles for the null distribution are 1.224 for $\alpha=0.1,1.358$ for $\alpha=0.05$ and 1.628 for $\alpha=0.01$.

After rejecting the null hypothesis, it is often of interes to know at which point in time the variance change happens. An intuitive estimator is the point $T^{*}$ in which the weighted difference between the estimated variances takes its maximum, i.e.

$$
T^{*}=\arg \max _{1 \leq j \leq T}\left|\hat{D} \frac{j}{\sqrt{T}}\left([\operatorname{Var} X]_{j}-[\operatorname{Var} X]_{T}\right)\right| .
$$


Wied et al. [2011] study the asymptotic local power of the test which is always larger than or equal to $\alpha$ under certain conditions. In Monte Carlo studies, Wied et al. [2011] investigate the power for different sample sizes and different variances in the first and second half of the time series. They set the variance in the left half to 1 and in the second half to 2, 4, 0.5 and 0.25. The empirical power reaches values at least of 0.886 already for a sample size of 800 . It is basically observed that the power increases with the sample size. Afterwards, Wied et al. [2011] apply the fluctuation test to a real financial data (different indices) and derive a portfolio strategy on the basis of their fluctuation test which they show to perform well in practice.

In the next section we apply the fluctuation test to a data set raised from a thermal spraying process. Before we can use the test, we have to remove trends and jumps in order to fulfill stationarity. Thus a time series filter is required which depends on a proper window width. Currently, it is not known how robust or sensitive the fluctuation test is against different choices of window widths. Therefore, in the next subsection we will use a filter for different window widths in order to study the sensitivity of the test.

\subsection{Application to thermal spraying process}

The time series from the thermal spraying process example contain trends as well as jumps. Thus a filter is required that is designed to detect both trends and jumps. We use the hybrid repeated median filter [Fried et al., 2006] which is implemented in Fried et al. [2012] and can be called by the function hybrid.filter() in $\mathrm{R}$ [R Core Team, 2012]. Figure 1 presents an exemplary application of the repeated median hybrid filter to the temperature time series for the situation Failure of one powder feeder. The left hand plot shows the real time series and the red line indicates the estimated signal by means of the hybrid repeated median filter. We observe that both the trend and the jump are detected very fast and the corresponding residuals on the right hand plot appear to be stationary.

We proceed as follows:

1. For each window width $(11,21,31, \ldots, 201)$ we proceed as follows:

a) For each situation (see Table 2) and for each particle property (temperature, velocity, flame width, flame intensity) we apply the hybrid repeated median filter

b) We subtract the real signal from the estimated signal in order to get residuals in order to remove trends and jumps 
Hybrid Filter

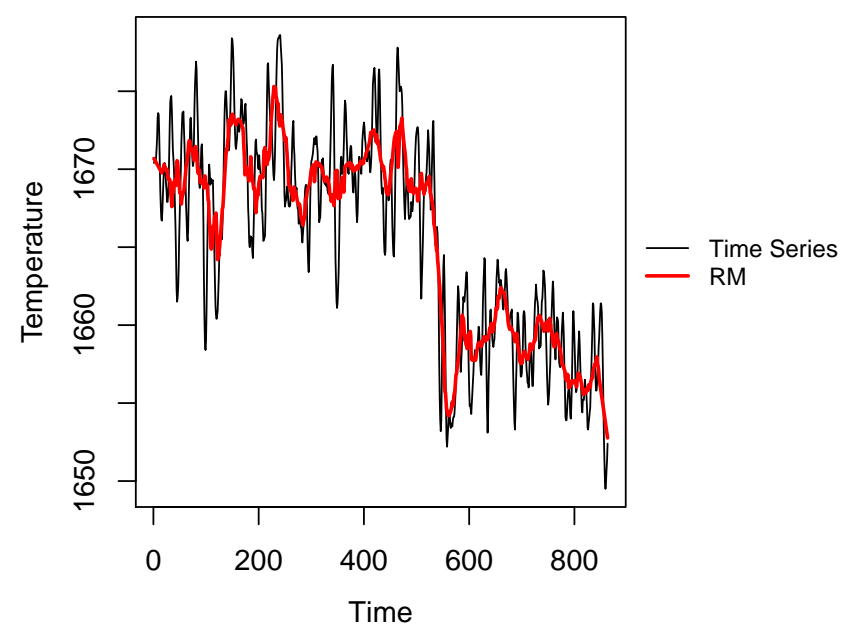

Hybrid filter

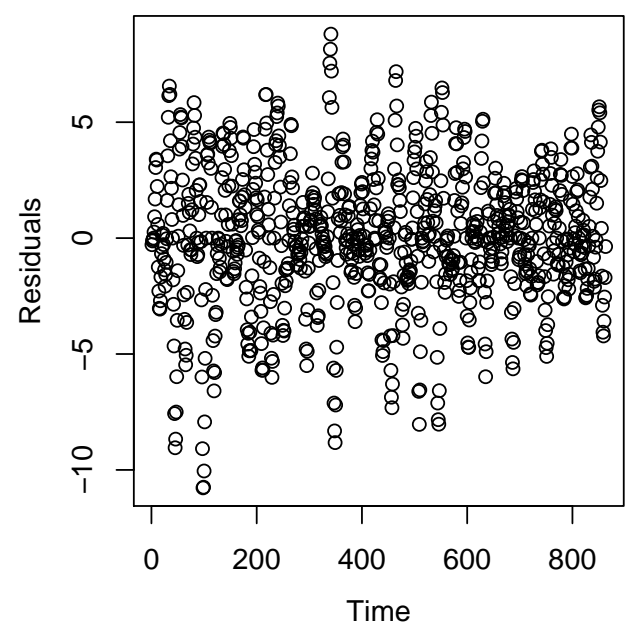

Figure 1: Application of the hybrid repeated median filter to temperature time series for situation Failure of one powder feeder

c) We calculate the test statistic for the fluctuation test

2. We plot the test static values against the corresponding window width

The resulting plots are displayed in Figures 3 and 2. The horizontal light blue line indicates the 0.95-quantile of the null distribution. It can be observed that if the test is statistically significant for the 0.05-level, then the result is consistent for nearly every situation. Thus, the statistical significance result appears to be independent of the window width except in the case of pressure drop of the carrier gas in Figure 2. On the right hand plot in Figure 2 the results for flame width are ambigous. While the first two test statistic values are not statistically significant, the rest of the values are greater than the 0.95-quantile. Nevertheless, the test statistic values exhibit non linear structures which appear to be very regular. On the left hand plot in Figure 3 the test statistic values stabilize after window width of 51 for temperature and velocity. The values for intensity are fairly constant for every window width. In the case of flame width, the values are slightly under the 0.95-quantile and decrease after a window width of 101. The only throughout statistically significant result is obtained for flame width on the the left hand plot in Figure 2. Here, every test statistic value is larger than the 0.95-quantile. The test statistic values for velocity tend to have an increasing structure except the first two. The values for temperature and intensity seem to stabilize. On the right hand plot in Figure 3 

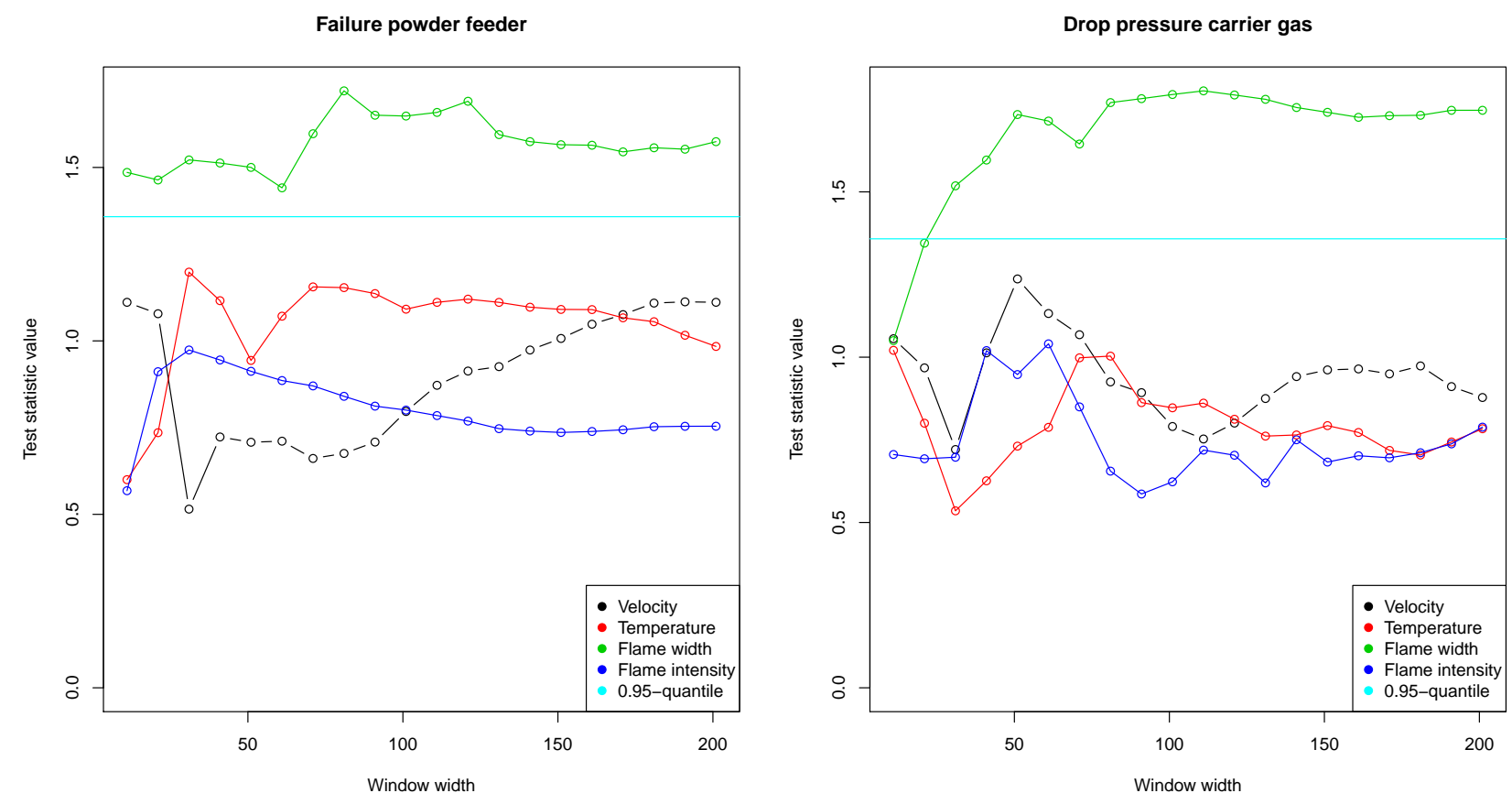

Figure 2: Robustness study for situations failure of one powder feeder and pressure drop of the carrier gas

we can also observe relatively regular structure except for window width. 

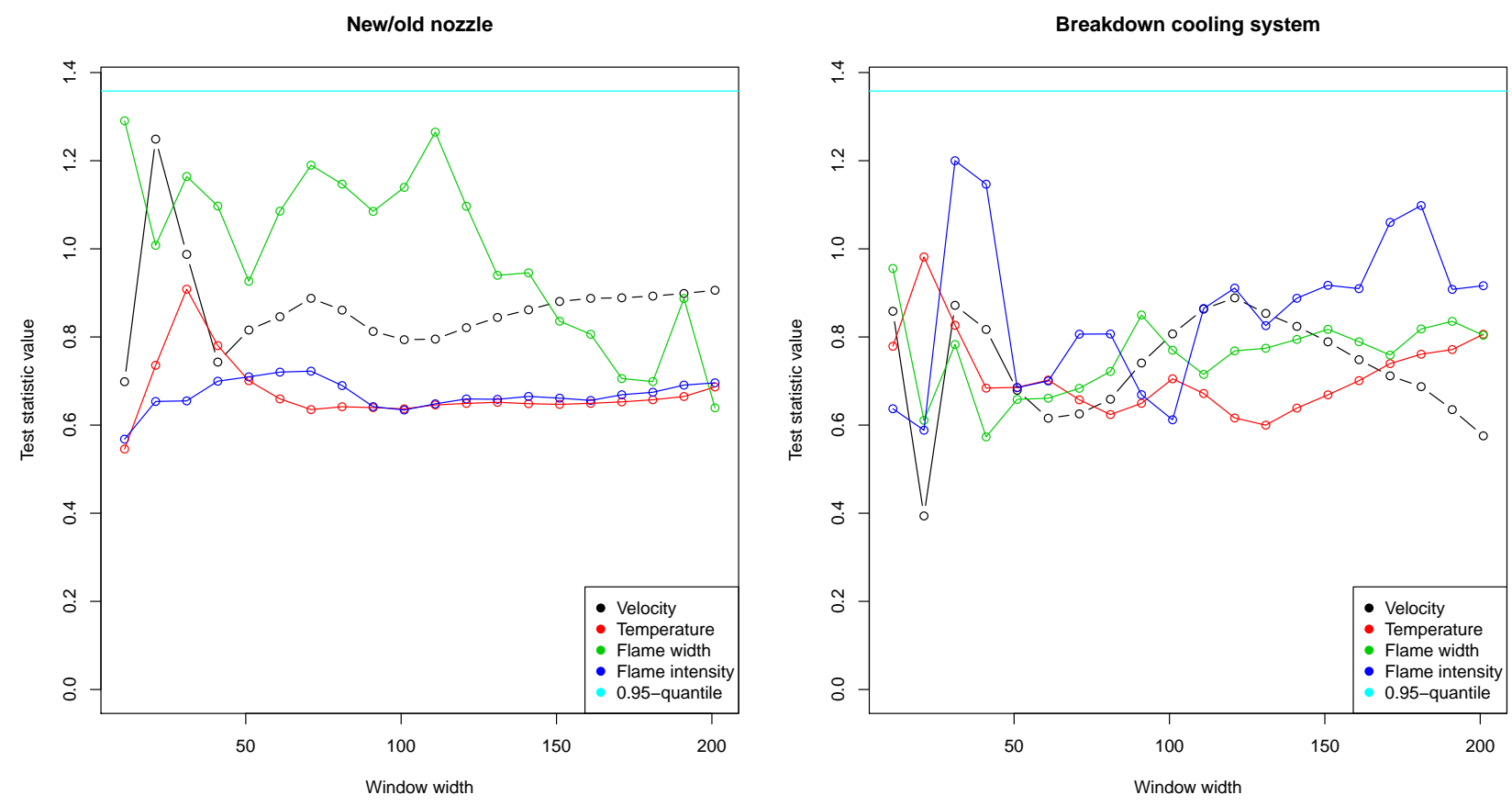

Figure 3: Robustness study for situations new/old nozzle and breakdown of AccuraSpray cooling

\section{Real-time detection of jumps and trend changes}

Under the assumption that the time series observations come from an unknown signal which is interrupted by noise and outliers, the Slope Comparing Adaptive Median [SCARM, Borowski and Fried, 2011] is a procedure for online signal extraction (i.e. smoothing) from nonstationary data stream time series. The signal carrying the relevant information is estimated in a moving time window, whose width is adapted to the current data situation at each time point $t$. This is done by means of a test for local linearity of the signal. This test offers good power for jumps (also called level shifts or edges) and trend changes, and can therefore be used for the online detection of such irregularities in thermal spraying processes.

\subsection{The SCARM-test}

As mentioned above, the SCARM assumes the observations $x_{t}$ of the time series $\left(x_{t}\right)_{t \in \mathbb{Z}}$ to come from

$$
X_{t}=\mu_{t}+\varepsilon_{t}+\eta_{t}
$$

where $\mu_{t}$ denotes the unknown underlying signal which is smooth but may exhibit sudden trend changes and level shifts. Furthermore, $\varepsilon_{t}$ is an error process consisting of independent 
random variables with zero expectation and time-dependent variance $\sigma_{t}^{2}$, and $\eta_{t}$ is an outlier process that occasionally generates large absolute values but is zero most of the time. The noise variance may slowly change over time, but is treated locally as almost constant. It is furthermore assumed that the signal is approximately linear in a certain window of size $n$ :

$$
\mu_{t-n+i} \approx \mu_{t}+\beta_{t} \cdot(i-n), \quad i=1, \ldots, n
$$

Under this assumption of local linearity, the SCARM estimates $\mu_{t}$, i.e. the signal at time $t$, by fitting a robust Repeated Median [RM, Siegel, 1982] regression line to the recent $n$ observations $x_{t-n+1}, \ldots, x_{t}$ :

$$
\hat{x}_{t-n+i}=\hat{\mu}_{t}+\hat{\beta}_{t} \cdot(i-n), \quad i=1, \ldots, n,
$$

where $\hat{\beta}_{t}$ is the slope and $\hat{\mu}_{t}$ the level at the rightmost design point $t$, which is the current time point in an online application. The level $\hat{\mu}_{t}$ is then the estimate of the signal $\mu_{t}$. Given the sample $\mathbf{x}_{t}:=\left(x_{t-n+1}, \ldots, x_{t}\right)$, the RM estimates $\hat{\beta}_{t}$ and $\hat{\mu}_{t}$ are

$$
\begin{aligned}
& \hat{\beta}_{t}:=\hat{\beta}\left(\mathbf{x}_{t}\right)=\operatorname{med}_{i \in\{1, \ldots, n\}}\left\{\operatorname{med}_{j \neq i}\left\{\frac{x_{t-n+i}-x_{t-n+j}}{i-j}\right\}\right\}, \\
& \hat{\mu}_{t}:=\hat{\mu}\left(\mathbf{x}_{t}\right)=\operatorname{med}_{i \in\{1, \ldots, n\}}\left\{x_{t-n+i}-\hat{\beta}_{t} \cdot(i-n)\right\} .
\end{aligned}
$$

The size $n$ of the window sample $\mathbf{x}_{t}$ is chosen automatically by the SCARM at each time $t$ according to the current data situation. This is done by means of a test for local linearity of the underlying signal $\mu_{t-n+1}, \ldots, \mu_{t}$ :

$$
\begin{array}{r}
\mathrm{H}_{0}: \mu_{t-n+i}=\mu_{t}+\beta_{t} \cdot(i-n), \quad i=1, \ldots, n, \\
\mathrm{H}_{1}: \mu_{t-n+i}=\left\{\begin{array}{l}
\mu_{t}^{\text {old }}+\beta_{t}^{\text {old }} \cdot(i-n), \quad i=1, \ldots, t_{0} \\
\mu_{t}^{\text {new }}+\beta_{t}^{\text {new }} \cdot(i-n), \quad i=t_{0}+1, \ldots, n
\end{array}\right.
\end{array}
$$

where $t_{0} \in\{1, \ldots, n-1\}$ and $\mu_{t}^{\text {old }} \neq \mu_{t}^{\text {new }}$ and $/$ or $\beta_{t}^{\text {old }} \neq \beta_{t}^{\text {new }}$. If $\mathrm{H}_{0}$ cannot be rejected, it is justifiable to approximate the signal by a regression line. The alternative $\mathrm{H}_{1}$ means that a level shift and/or trend change takes place in the time window of size $n$. In this case the width of the window is set down to a small value in order to estimate the signal exclusively on the observations coming after the change, see Borowski and Fried [2011]. However, since we are interested in detecting changes rather than adapting window widths, we do not introduce the whole SCARM algorithm. Instead we only explain the test procedure as it can be used to detect signal changes in real-time.

The SCARM divides the window sample $\mathbf{x}_{t} \in \mathbb{R}^{n}$ into two separate samples, a left-hand and 
right-hand sample $\mathbf{x}_{t}^{\text {left }}=\left(x_{t-n+1}, \ldots, x_{t-r}\right) \in \mathbb{R}^{\ell}$ and $\mathbf{x}_{t}^{\text {right }}=\left(x_{t-r+1}, \ldots, x_{t}\right) \in \mathbb{R}^{r}$ with $n=\ell+r$. The referring random vectors are $\mathbf{X}_{t}^{\text {left }}$ and $\mathbf{X}_{t}^{\text {right }}$. Furthermore, let $\hat{\beta}_{t}^{\text {right }}:=\hat{\beta}\left(\mathbf{X}_{t}^{\text {left }}\right)$ and $\hat{\beta}_{t}^{\text {left }}:=\hat{\beta}\left(\mathbf{X}_{t}^{\text {right }}\right)$ denote the left-hand and right-hand $R M$ slopes. Then the test statistic of the SCARM $T_{\text {SCARM }}$ is

$$
T_{\text {SCARM }}:=\frac{D_{t}}{\sqrt{\widehat{\operatorname{Var}}\left(D_{t}\right)}},
$$

where $\widehat{\operatorname{Var}}\left(D_{t}\right)$ is an estimator of the variance of the slope difference $D_{t}:=\hat{\beta}_{t}^{\text {right }}-\hat{\beta}_{t}^{\text {left }}$ which is explained below. Borowski and Fried [2011] reject $\mathrm{H}_{0}$ if $\left|T_{\mathrm{SCARM}}\right|$ is larger than the $1-\alpha / 2$ quantile of the $t_{f}$-distribution, where the degrees of freedom $f=f(\ell, r)$ depend on the sample sizes $\ell$ and $r$, and $\alpha$ is the level of the statistical significance. To estimate $\operatorname{Var}\left(D_{t}\right)$ under $\mathrm{H}_{0}$, Borowski and Fried use that under certain conditions

$$
\operatorname{Var}\left(D_{t}\right)=\sigma_{t}^{2} \cdot[\mathrm{V}(\ell, 1)+\mathrm{V}(r, 1)]
$$

Here $\mathrm{V}(n, 1)$ denotes the variance of the RM slope for samples of size $n$ with errors coming from a specified distribution. Borowski and Fried obtain approximations $v_{n}:=\hat{\mathrm{V}}(n, 1)$ by means of simulations and estimate $\sigma_{t}$ using the model-free and robust scale estimator $Q^{\text {adj }}$ [Gelper et al., 2009]. The $Q^{\text {adj }}$ is applied directly to the data and does not require a preceding regression fit. This leads to a gain in power of the SCARM-test since large estimations of $\operatorname{Var}\left(D_{t}\right)$ are prevented [Borowski and Fried, 2011]. Given a sample $\mathbf{x}_{t}$, the $Q^{\text {adj }}$ scale estimate of the noise scale is

$$
\hat{\sigma}_{t}=Q_{\delta}^{\mathrm{adj}}\left(\mathbf{x}_{t}\right)=c_{n} \cdot h_{(t-n+\lfloor\delta(n-2)\rfloor)},
$$

where $h_{(t-n+\lfloor\delta(n-2)\rfloor)}$ is the $\delta$-quantile of the vertical heights $h_{t-n+i}, i=2, \ldots, n-1$, of the $n-2$ triangles built by each triple of adjacent observations $x_{t-n+i-1}, x_{t-n+i}, x_{t-n+i+1}$ :

$$
h_{t-n+i}=\left|x_{t-n+i}-\frac{x_{t-n+i-1}+x_{t-n+i+1}}{2}\right| .
$$

The factors $c_{n}$ ensure the unbiasedness of the estimator for samples of size $n$ with errors coming from a specified distribution and can be obtained by means of simulations. Gelper et al. [2009] suggest to choose $\delta=0.5$ to obtain reasonable robustness and efficiency of the $Q^{\text {adj }}$ estimator. Borowski and Fried obtain the constants $v_{n}$ and $c_{n}$ by means of simulations using standard normal errors. However, if the data are positively (negatively) autocorrelated, these constants are too small (too large), so that the proposed estimator

$$
\widehat{\operatorname{Var}}\left(d_{t}\right)=c_{n} \cdot h_{(t-n+\lfloor n / 2-1\rfloor)} \cdot\left(v_{\ell}+v_{r}\right) \text {, }
$$


has a downwards (upwards) bias [Borowski, 2013]. To tackle this problem, Borowski obtains constants $v_{n}^{\varphi}$ and $c_{n}^{\varphi}$ by means of simulations on data from an $\mathrm{AR}(1)$-process with parameter $\varphi \in\{-0.9,-0.6, \ldots, 0.9\}$ and standard normal error. The constants $v_{n}^{\varphi}$ and $c_{n}^{\varphi}$ can thus be chosen w.r.t. the parameter $\varphi$. Since the thermal spraying data exhibit moderate positive autocorrelations, we use $\varphi=0.6$.

\subsection{Application to the thermal spraying time series}

We apply the SCARM-test in a time window of fixed width $n=\ell+r$, using a slightly modified version of the $\mathrm{R}$ function scarm.filter from the $\mathrm{R}$ package robfilter [Fried et al., 2012, $\mathrm{R}$ Core Team, 2012]. The new $\mathrm{R}$ function, named scarm.detection, can be downloaded on the website http://www.statistik.tu-dortmund.de/1543.html. Application of the SCARMtest to the thermal spraying time series is done offline here, yet an online application would deliver the same results. It is mandatory to choose a small statistical significance level $\alpha$ as one
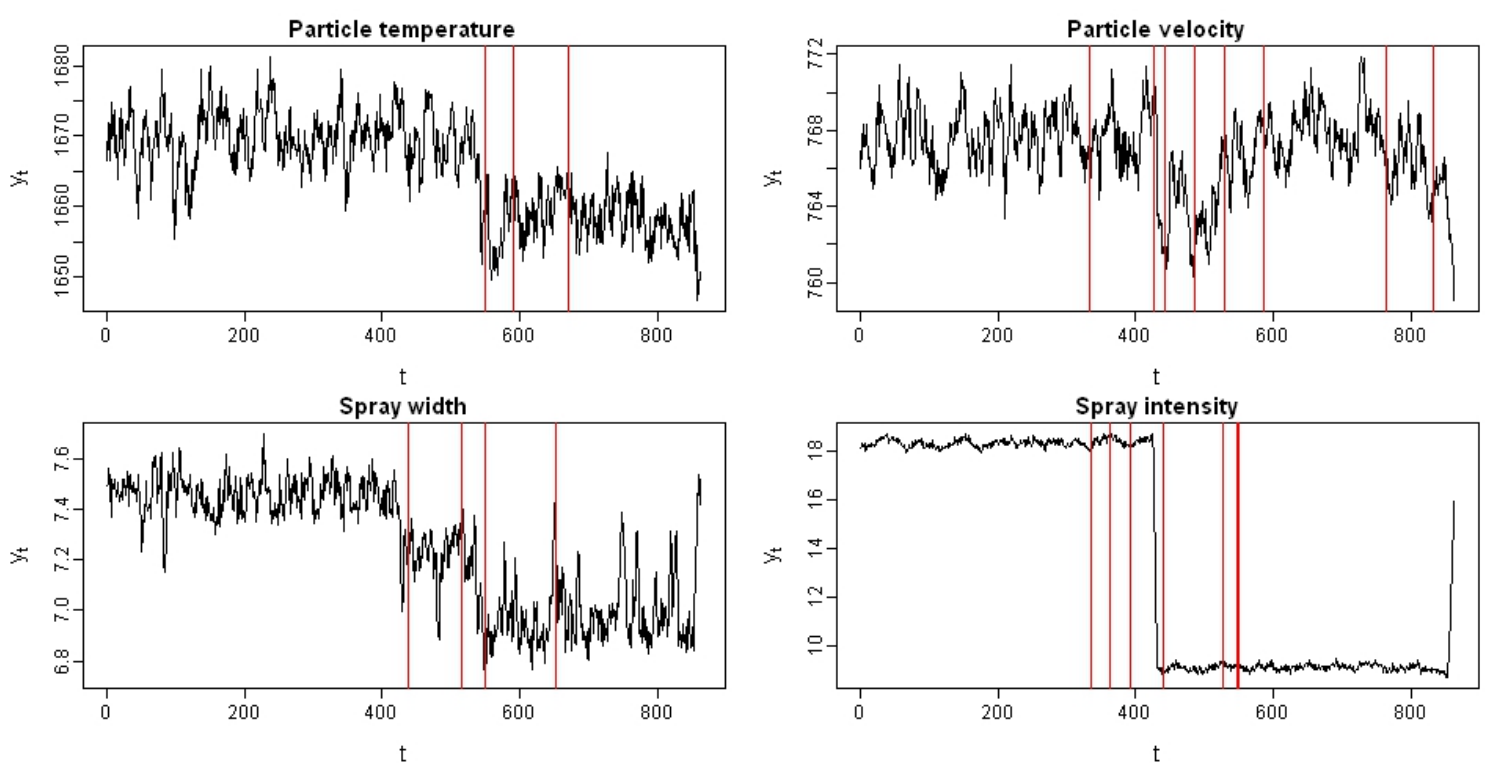

Figure 4: Time series of particle temperature, particle velocity, spray width and spray intensity in case of a failure of one powder feeder at $t=420$; signal changes detected by the SCARM-test are marked by red vertical lines.

expects one false detection every $1 / \alpha$ time points if $\mathrm{H}_{0}$ is true, i.e. if the signal is linear. Since [Borowski and Fried, 2011] find that the SCARM-test yields reasonable power also for small levels of statistical significance, we set $\alpha \hat{=}$ sign. level $=0.001$. Larger window widths $\ell$ and $r$ lead to a higher power of the test [Borowski and Fried, 2011]. However, signal changes are 
detected with a delay of approximately $r / 3$ time points, so that $r$ should not be too large. We therefore choose $(\ell, r)=(200,40)$, a rather large value for $\ell$ and a moderate value for $r$. Since
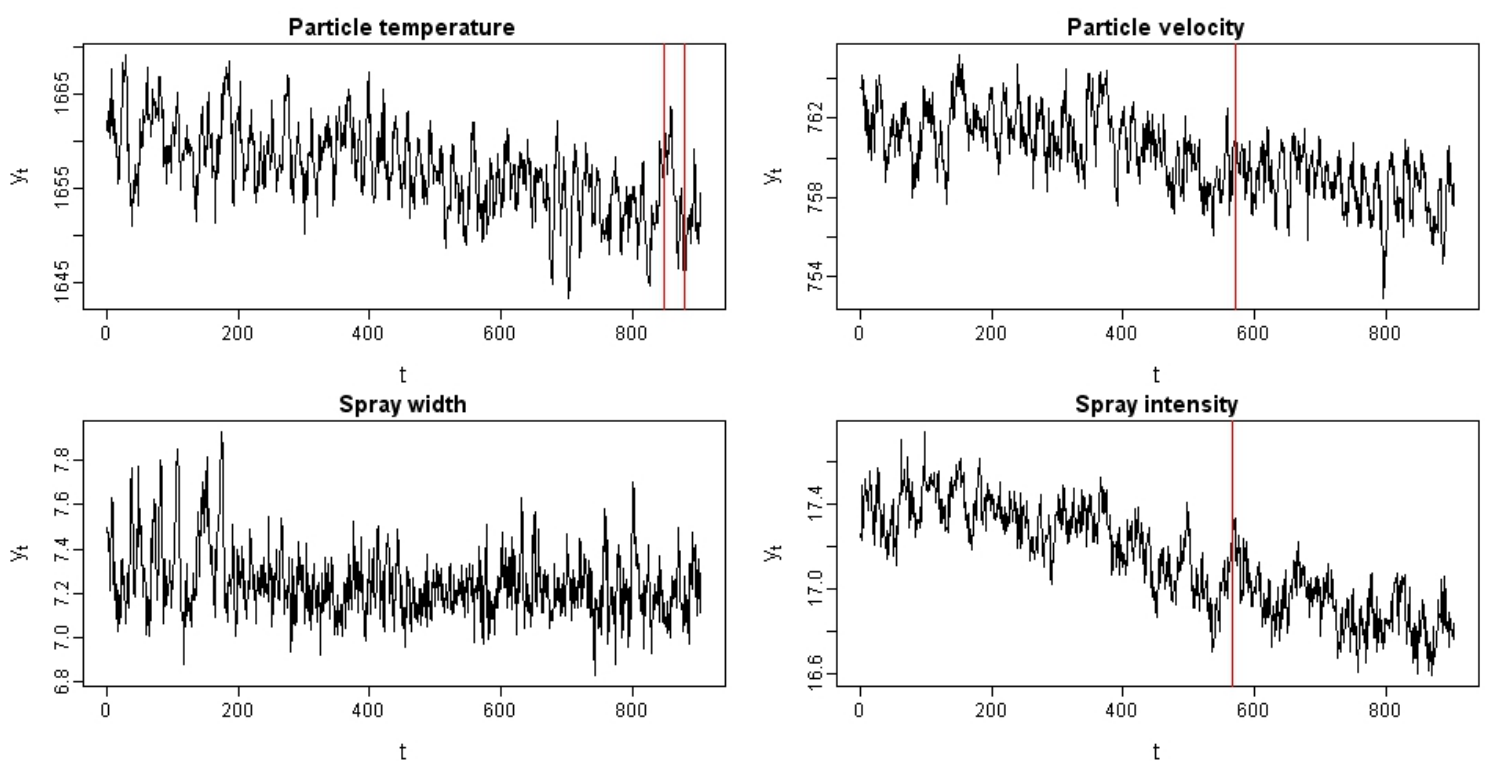

Figure 5: Time series of particle temperature, particle velocity, spray width and spray intensity in case of a gas pressure drop at $t=420$; signal changes detected by the SCARM-test are marked by red vertical lines.

the data are rounded to one decimal place, ties are likely to occur in the window samples, leading to a downward bias of the $Q^{\text {adj }}$ estimator. To overcome this problem, we 'wobble' the data [Fried and Gather, 2007] by adding white noise from a normal distribution with zero mean and variance $\varsigma^{2}$. For each time series, we choose $\varsigma$ equal to half of the empirical standard deviation of the first 400 observations $x_{1}, \ldots, x_{400}$, because the time series can be assumed to be (at least almost) stationary in this period. Due to the wobbling there are no more ties in the data, yet signal changes are not covered by noise since the error scale is small enough. Again we consider

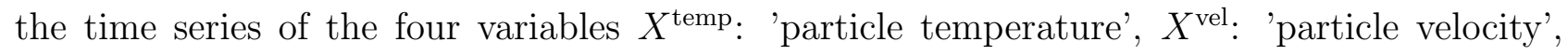
$X^{\text {wid. }}$ 'spray jet width', and $X^{\text {int: }}$ 'spray jet intensity' for the four cases listed in Table 1 . The Figures $4-7$ display the time series $\left(x_{t}^{\text {temp }}\right),\left(x_{t}^{\text {vel }}\right),\left(x_{t}^{\text {wid }}\right),\left(x_{t}^{\text {int }}\right)$ for each of the four cases. In each case the change happens at $t=420$. Signal changes detected by the SCARM-test are marked by red vertical lines.

Due to the failure of the powder feeder (Figure 4), jumps occur respectively around $t=420$ in the series of $X^{\mathrm{vel}}, X^{\mathrm{wid}}, X^{\mathrm{int}}$ and around $t=550$ in the series of $X^{\text {temp }}$. The SCARM-test detects each jump after only a short time. After the failure of the powder feeder at $t=420$, 

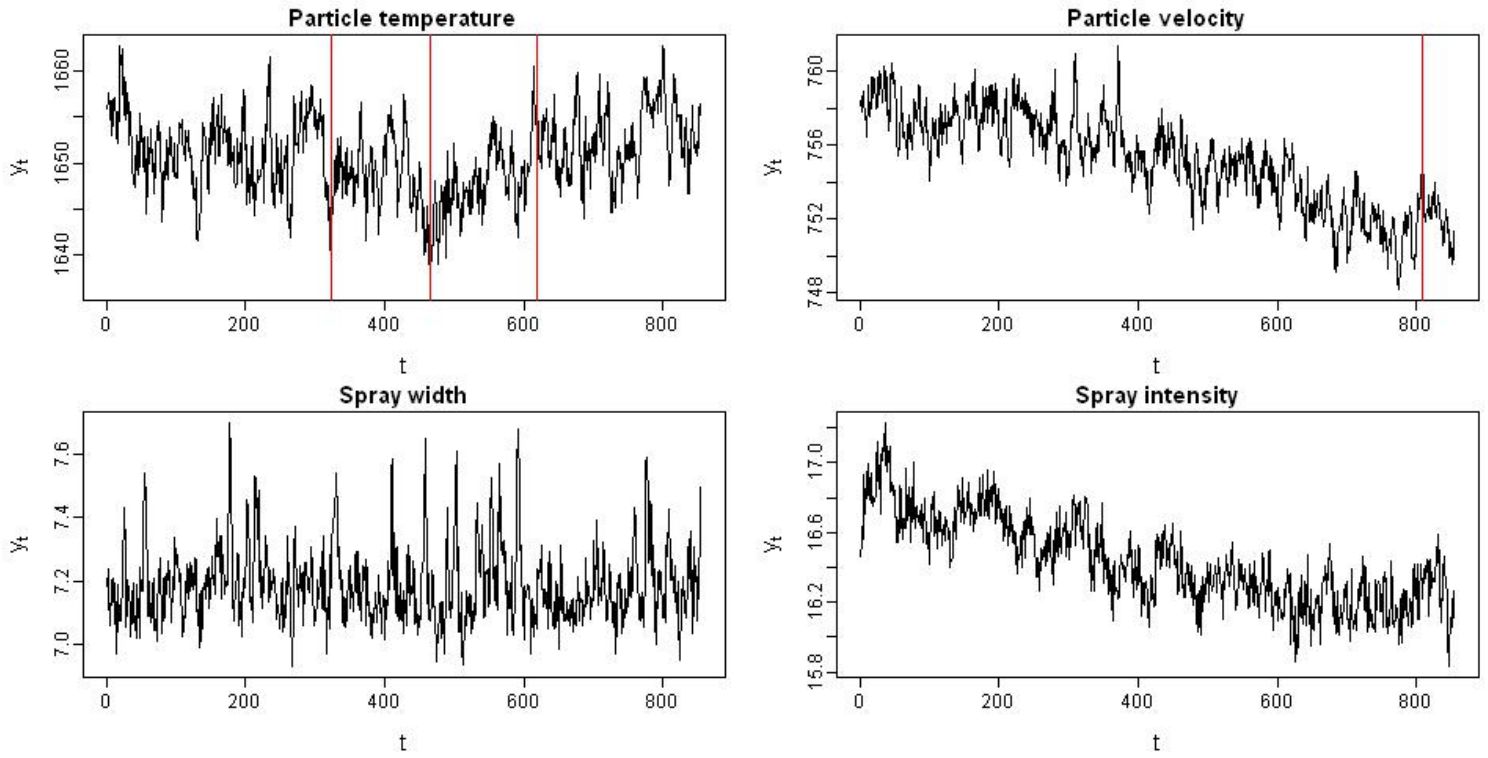

Figure 6: Time series of particle temperature, particle velocity, spray width and spray intensity in case of a failure of the cooling at $t=420$; signal changes detected by the SCARMtest are marked by red vertical lines.

the SCARM-test rejects the null hypothesis several times, which is an indication for the nonstationarity of the time series. The rejections at time points $t<420$ can be regarded as type I errors.

The gas pressure drop (Figure 5) at time $t=420$ does not lead to obvious jumps or trend changes in the four series. Hence, the SCARM does not detect any change around this time point. There are detections in the series $\left(x_{t}^{\mathrm{temp}}\right),\left(x_{t}^{\mathrm{vel}}\right)$, and $\left(x_{t}^{\mathrm{int}}\right)$ at later time points, but these are rather false than true detections in our opinion.

The four time series for the case 3: 'failure of the cooling system' are displayed in Figure 6. Here only the time series of $X^{\text {temp }}$ offers a visible signal change (around $t=450$ ), and this change is detected by the SCARM. The other time series do not show obvious changes, and the SCARM does not detect any signal change. However, there are some detections because of minor fluctuations, which can be regarded as type I errors of the test procedure.

The time series for the case 4: 'old vs new nozzle' are shown in Figure 7 . The new nozzle leads to distinct changes in the four time series around $t=420$, which are detected by the SCARM-test in each case. There are several additional changes detected in the series $\left(x_{t}^{\mathrm{vel}}\right)$ and $\left(x_{t}^{\text {int }}\right)$ after $t=420$. Similar to the case of a failure of one powder feeder, these detections indicate a nonstationary behavior of the series due to the change of the nozzle. Furthermore, 

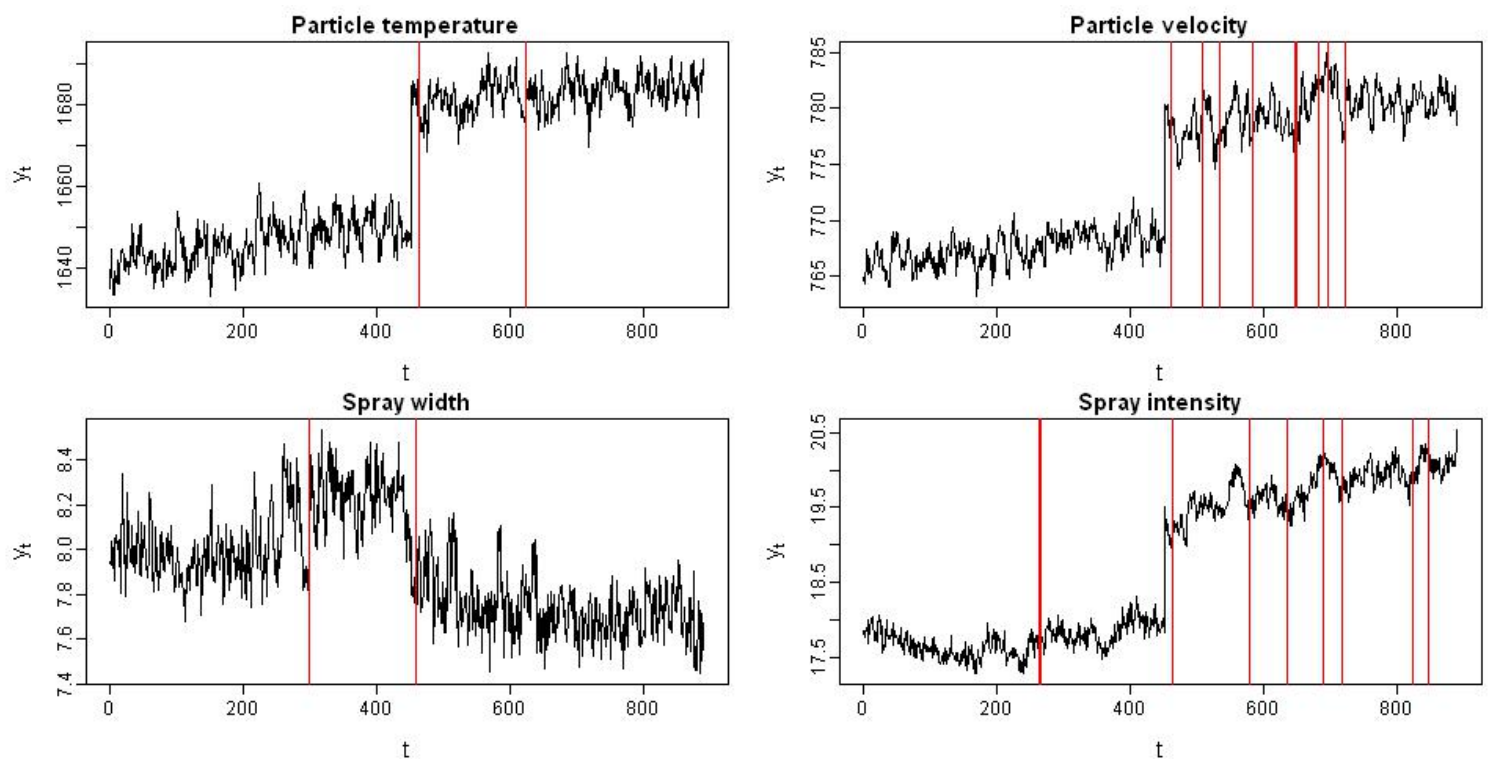

Figure 7: Time series of particle temperature, particle velocity, spray width and spray intensity in case of a change of the nozzle at $t=420$; signal changes detected by the SCARMtest are marked by red vertical lines.

in each of the the four time series a jump is visible to a greater or lesser extent around time $t=200$ to $t=250$. The distinct jumps in the series $\left(x_{t}^{\text {wid }}\right)$ and $\left(x_{t}^{\text {int }}\right)$ are detected, whereas the small jumps in $\left(x_{t}^{\text {temp }}\right)$ and $\left(x_{t}^{\mathrm{vel}}\right)$ are not detected. 


\section{Discussion}

We investigated the possibilities and limits of recently developed methods to find structural changes in time series of thermal spraying processes: a fluctuation test by Wied et al. [2011] for the off-line detection of changes in the variance and the SCARM-test by Borowski and Fried [2011] for the on-line detection of jumps and trend changes. Since the thermal spraying processes were manipulated by the experimenter, the time points of the changes are known.

Before calculating the test statistic of the fluctuation test we applied the hybrid repeated median filter. This procedure was repeated for several different window widths in order to study the robustness of the test against a choice of window width. The study exhibits that the result of statistical significance appears to be independent of the window width, except for one special case for flame width in the situation failure of one powder feeder. Here, the test was significant for almost all window widths except the first two. However, the test statistic values plotted against the corresponding window width posses a nonlinear but regular structure.

The application of the SCARM-test to the thermal spraying time series shows both the merits and limits of the SCARM-test. The procedure is useful for the detection of visible jumps and trend changes but rather not for minor structural changes. However, it works in real-time, so that the engineer can react immediately to structural breaks. Furthermore, the SCARMtest is appropriate to nonstationary time series and robust against outliers. As a result, a SCARM-based alarm system for the online-surveillance of spraying processes is both feasible and beneficial: when the SCARM-test detects a change, an alarm is given and the engineer can react.

\section{Acknowledgements}

The financial support of the Deutsche Forschungsgemeinschaft (SFB 823, projects A1, B1 and C1) is gratefully acknowledged. 


\section{References}

M. Borowski. Echtzeit-Extraktion relevanter Information aus multivariaten Zeitreihen basierend auf robuster Regression. Dissertation, Fakultät Statistik, Technische Universität Dortmund (in German), 2013.

M. Borowski and R. Fried. Robust repeated median regression in moving windows with dataadaptive width selection. Discussion Paper 28/11, SFB 823, Technische Universität Dortmund, 2011.

R. Fried and U. Gather. On rank tests for shift detection in time series. Computational Statistics and Data Analysis, 52:221-233, 2007.

R. Fried, T. Bernholt, and U. Gather. Repeated median and hybrid filters. Computational Statistics and Data Analysis, 50:2313-2338, 2006.

R. Fried, K. Schettlinger, and M. Borowski. robfilter: Robust Time Series Filters, 2012. URL http://CRAN.R-project.org/package=robfilter. R package version 3.1.

S. Gelper, K. Schettlinger, Ch. Croux, and U. Gather. Robust online scale estimation in time series: a model-free approach. Journal of Statistical Planning and Inference, 139:335-349, 2009.

R Core Team. R: A Language and Environment for Statistical Computing. R Foundation for Statistical Computing, Vienna, Austria, 2012. URL http://www.R-project.org/. ISBN 3-900051-07-0.

A.F. Siegel. Robust regression using repeated medians. Biometrika, 69(1):242-244, 1982.

D. Wied, M. Arnold, N. Bissantz, and D. Ziggel. A new fluctuation test for constant variances with applications to finance. Metrika, 2011. Published online: 06 November 2011. 


